\title{
ECLAMPSIA DEL PUERPERIO *
}

\section{Doctores Fernando Sánchez Torres \$:, Hugo Guevara Jaramillo $*$, Jesús A. Gómez Palacino *\%}

Cuando un paciente que tuvo manifestaciones de preeclampsia durante su embarazo padece crisis convulsivas generalizadas después de expulsada la placenta, ha de pensarse, en principio, que tiene una eclampsia del puerperio o del postparto. Lo que aún no se ha establecido es hasta cuántos días después del parto puede aceptarse que esas convulsiones sean ocasionadas evidentemente por la toxemia gravídica. Tatum, en su clasificación de la toxemia, admite que la eclampsia puede aparecer dentro de los primeros catorce días del puerperio (I2), basado en que él observó un caso al décimoquinto día y que, al parecer, se debió a un síndrome de toxemia iniciado después del parto (II). Canillas (I) incluye uno en el vigésimonoveno día y Winterton, citado por Tatum (12), otro a los 26 días. Samuels (7) comunicó de una paciente que padeció convulsiones 23 días después de su parto y Steiner y Plasha (io) relataron un caso observado al décimosexto día Aunque en todos los casos señalados atrás faltó la comprobación mediante la biopsia renal de que se trataba, en efecto, de una toxemia gravídica, la evolución clínica y los exámenes de laboratorio hacen presumir que realmente lo fueron.

Si convenimos, como hasta ahora lo ha sido en forma unánime, que la toxemia gravídica es un síndrome relacionado íntimamente con la presencia activa de la placenta, es difícil entender por qué una mujer que no está ya embarazada pueda exhibir manifestaciones clínicas de preeclampsia y aun llegar a padecer convulsiones. Es natural, entonces, que la eclampsia del puerperio contribuya a hacer todavía más confusos los conceptos acerca de la etiología y la fisiopatología de la toxemia gravídica. Así como es posible descartar la participación del feto y del líquido amniótico en la aparición de la preeclampsia-eclampsia al observar este síndrome en los casos de mola hidatiforme, la ocurrencia de eclampsia aparecida tardíamente durante el curso

* Trabajo elaborado en el Servicio de Toxemias del Instituto Materno Infantil, de Bogotá.

* De la Sección de Obstetricia y Ginecología (Universidad Nacional). 
del puerperio, obliga a pensar que además de la placenta pueden existir otros factores - hasta el momento ignorados- que favorecen o conducen a la aparición de la toxemia. Además, ese hecho resta importancia a la distensión uterina o al reflejo útero-renal como elementos desencadenantes o coadyuvantes de la enfermedad.

El motivo de esta comunicación es dar a conocer algunos aspectos del problema, basados en la observación cuidadosa de 64 casos de eclampsia del puerperio.

\section{Material y método}

Los 64 casos de eclampsia del puerperio, material de este estudio, corresponden a pacientes que fueron atendidas en el Servicio de Toxemias del Instituto Materno Infantil "Concepción Villaveces de Acosta", de Bogotá, durante un lapso de seis años ( I958-I963), tiempo durante el cual fuer on observados 430 casos de eclampsia, correspondiendo, pues, el $14.88 \%$ a la forma puerperal.

Se consideraron como eclampsias del puerperio todos aquellos casos de pacientes que presentaron crisis convulsivas gen ralizadas por primera vez, después de que se hubo verificado su parto. Se descartaron, por lo tanto, aquellos en que a pesar de apreciarse convulsiones en el curso del puerperio, su iniciación había tenido lugar antes o durante el trabajo de parto.

El manejo y tratamiento de estas enfermas fue más o menos el mismo y consistió en colocarlas en un ambiente alejado de los estímulos externos, en procurar una buena ventilación pulmonar y en la aplicación simultánea de fenobarbital, sulfato de magnesio y acepromazina (Plegicil). Además, durante 24 a 36 horas fueron sometidas a privación hídrica, es decir, no recibieron ninguna clase de líquidos por ninguna vía.

Veinticuatro mujeres habían tenido el parto en su domicilio y fueron conducidas al hospital por haber presentado convulsiones después de aquél Cuarentitrés ingresaron a la maternidad en trabajo de parto y luego que aparecieron las convulsiones fueron trasladadas al Servicio de Toxemias Finalmente, sólo una paciente inició su eclampsia estando ya hospitaliza da en el servicio. Se advirtió hipertensión arterial antes del parto en I7 mujeres, en 23 la presión era normal y en 24 no fue posible obtener este dato pues ya eran puérperas cuando ingresaron al hospital. No hubo evidencia clínica de edema en 27 pacientes.

En 57 casos el parto se había verificado de manera espontánea, sin anestesia; en 5 se hizo aplicación de fórceps, 4 con bloqueo de los nervios pudendos y a con bloqueo en silla; por último, 2 pacientes fueron sometidas a cesárea, practicadas ambas con anestesia raquídea. Sesentidós niños nacieron vivos (un parto gemelar) y 3 nacieron muertos.

Edad de las pacientes. $22(34.3 \%)$ eran menores de I9 años y Io $(15.6 \%)$ eran mayores de 30 años. 
Paridad. De las 64 enfermas, 33 eran primíparas $(5 \mathrm{I} .6 \%)$, I8 eran secundíparas $(28.1 \%$ ) y 13 completaban 3 ó más partos $(20.3 \%)$.

Edad de la gestación. En 16 oportunidades no fue posible precisar el dato de la última menstruación. Veinticinco $(39 \%)$ tuvieron su parto luego de una gestación de término. El embarazo terminó de 36 a 38 semanas en 15 mujeres $(22.8 \%)$ y solamente en un caso fue inferior a 28 semanas.

Control prenatal. Unicamente 2 mujeres tuvieron control prenatal adecuado.

Medicación postparto. Hubo certeza de que 22 enfermas habían recibido maleato de ergonovina por vía parenteral u oral, antes de que apareciera la eclampsia. Se había aplicado ocitocina en 8 oportunidades y fenobarbital en 3. En los demás casos no se había administrado ninguna medicación o no fue posible identificarla.

Tiempo entre el parto y la aparación de la eclampsia:

\begin{tabular}{lr} 
I5 minutos & I I casos \\
Media a 2 horas & 8 casos \\
2 a 4 horas & Io casos \\
4 a 8 horas & I6 casos \\
8 a I2 horas & 6 casos \\
I2 a 24 horas & 6 casos \\
36 horas & I caso \\
2 días & I caso \\
4 días & I caso \\
9 días & I caso \\
Sin dato & 3 casos \\
\hline
\end{tabular}

Como vemos, en 57 oportunidades $(90 \%)$ la eclampsia apareció en el curso de las primeras 24 horas del puerperio. Creemos de interés presentar, muy sucintamente, las historias clínicas de las pacientes que padecieron convulsiones al cuarto y noveno días de puerperio.

El primer caso (Historia $\mathrm{N}^{\circ} 728 \mathrm{I} 3$ ) corresponde a una enferma de 26 años, $\mathrm{G}_{4} \mathrm{P}_{3}$, con una gestación que cursaba en la semana 35. Ingresó en estado avanzado de anemia carencial e insuficiencia cardíaca, con presión arterial de $135 / 60$, edema generalizado, indicios de proteínas en la orina y sin cambios en el fondo de ojo. Se suministraron cardiotónicos y clorotiazida y se corrigió su anemia con transfusiones repetidas. A los 20 días de su ingreso, y sin haber tenido manifestaciones de toxemia, tuvo un parto espontáneo de un niño en buenas condiciones. Durante los primeros tres días del puerperio recibió maleato de ergonovina por vía oral. Al cuarto día padeció una crisis convulsiva generalizada encontrándose una presión arterial de 230/120 y una proteínuria de r.26 gr/oo. La eclampsia fue prontamente controlada y la paciente abandonó el servicio 8 días más tarde sin ninguna manifestación de toxemia. No asistió a control postnatal.

El segundo caso (Historia No I3716) pertenece a una paciente de 29 años, $\mathrm{G}_{5} \mathrm{P}_{4}$, en cuya anamnesis figuraba el antecedente de haber padecido convulsiones tres años antes, al octavo mes de su embarazo. Ingresó al hos- 
pital en trabajo de parto y se advirtió una presión arterial de $180 / 130$, edema de miembros inferiores e indicios de albúmina en la orina. Las arterias retinianas era muy estrechas y brillantes. El parto se verificó espontáneamente, naciendo una niña de 2.600 gm. Salió del hospital a los cinco días con presión arterial de 150/I00 y proteinuria negativa. Se hizo entonces diagnóstico de hipertensión crónica con toxemia sobreagregada. Cuatro días después, es decir al noveno día del puerperio, reingresó luego de haber padecido en su casa tres crisis convulsivas generalizadas y al llegar al hospital hizo un cuarto ataque. La presión arterial era de 170/130, no se apreció edema y la proteinuria fue de $0.420 \mathrm{gr} / \mathrm{oo}$. El fondo de ojo no mostró cambios distintos a los observados la primera vez. La eclampsia se controló fácilmente y fue dada de alta 20 días más tarde, después de haberla estudiado detenidamente y haber concluído que se trataba de una hipertensión crónica de causa indeterminada (esencial)

Número de crisis antes del tratamiento:

\begin{tabular}{llr}
\hline I crisis & 24 casos \\
2 crisis & I9 casos \\
3 crisis & 9 casos \\
4 crisis & 7 casos \\
5 crisis & I caso \\
8 crisis & 2 casos \\
20 crisis & I caso \\
\hline
\end{tabular}

Una vez que ingresó cada paciente al Servicio de Toxemias, y antes de practicar cualquier tipo de examen, se aplicaron $400 \mathrm{mg}$. de fenobarbital, 20 cc. de sulfato de magnesio al $20 \%$ y $4 \mathrm{mg}$. de acepromazina. Media hora más tarde se procedió al examen clínico y se tomó una muestra de orina. En estas condiciones se encontró que 53 pacientes presentaban hipertensión arterial (más de $90 \mathrm{~mm}$. de $\mathrm{Hg}$ de presión mínima) y i I tenían una presión normal. En $27(42 \%)$ hubo evidencia clínica de edema y en 36 $\left(5^{6 \%}\right)$ la proteinuria fue dosificable.

Número de crisis después del tratamiento:
Ninguna

I crisis

2 crisis

4. crisis
$53 \operatorname{casos}(83 \%)$

6 casos

4 casos

I caso
Transcurridas 24 horas desde el último ataque convulsivo, se consideró la eclampsia controlada. Entonces se suministró únicamente fenobarbital por vía oral y se procedió a estudiar cada paciente para tratar de precisar el diagnóstico.

Morbilidad. Dos mujeres sufrieron accidente cerebrovascular hemorrágico, del cual se recuperaron posteriormente. Una sufrió luxación del maxilar inferior durante las crisis convulsivas, y otra luxación de la articulación del hombro.

Mortalidad. De las 64 pacientes fallecieron $2(3.1 \%)$, ambas por hemo- 
rragia cerebral. El primer caso fue el de una primípara de $2 \mathrm{I}$ años que padeció uno sola crisis convulsiva tres horas después de haber tenido un parto espontáneo. La autopsia confirmó el diagnóstico de toxemia y puso de presente una hemorragia intraventricular masiva. El segundo caso correspondía a una secundípara de 32 años que tuvo su parto en su domicilio y que pocos minutos después inició un verdadero estado epiléptico, pues al llegar al hospital había presentado 20 crisis convulsivas. En el servicio no repitió las convulsiones pero se advirtió la ocurrencia de una hemorragia cerebral, falleciendo algunas horas más tarde. La autopsia mostró lesiones viscerales de toxemia gravídica, junto con cambios de pielonefritis crónica. Además, la muerte fue la consacuencia de una severa hemorragia cerebral con inundación ventricular.

Control posterior. Sólo fue posible controlar hasta por 3 meses a $2 \mathrm{I}$ de las 62 enfermas. Seis de ellas continuaban con hipertensión arterial. Luego del estudio de cada paciente se llegó a la conclusión de que 5I sufrían de toxemia aguda y ${ }_{3} 3$ agregada a un proceso crónico vascular hipertensivo.

\section{COMENTARIO}

La mujer que ha tenido preeclampsia durante su embarazo no es raro que presente complicaciones derivadas de su enferm:dad en el curso del puerperio. Una de estas complicacio- nes es el estado convulsivo generalizado, que puede aparecer también sin que se hayan advertido signos de toxemia en el transcurso del embarazo y del parto mismo. De un grupo de 95 pacientes que padecieron convulsiones en el puerperio - y que corresponden a los casos comunicados por Tatum (12), Stander (9) y Samuels (7)-, el $57 \%$ no manifestó previamente evidencia clínica de toxemia eclamptogénica. Del grupo de 64 enfermas estudiadas por nosotros, en 40 se había practicado examen clínico durante el parto y solamente en i7 se comprobó hipertensión arterial. Este hecho sugiere la posibilidad de que la toxemia se manifieste clínicamente algunas horas después del parto, y hasta algunos días más tarde.

La imprevista e inexplicable aparición extemporánea de la eclampsia contraría mucho concepto tenido como cierto, acerca de la etiología de la toxemia gravídica. Es axiomático afirmar que sin placenta no hay toxemia, queriendo significar con esto que es ese órgano transitorio el que provoca la aparición del síndrome de preeclampsia-eclampsia. Todos los estudios llevados a cabo confirman este hecho, pero al abordar el problema de la eclampsia del puerperio pierde mucho de su validez. Aunque se ha querido sostener que la toxemia manifestada en el postparto es debida a la persistencia en la cavidad uterina de alguna porción de placenta por causa de un alumbramiento incompleto (3), el hecho de que los signos de toxemia cedan fácilmente al trata- 
miento médico - sin recurrir al raspado endouterino- indica que tal teoría carece de suficiente peso. Además, de ser así, ¿ por qué cuando estaba funcionando toda la placenta no se hizo evidente la preeclampsia-eclampsia? Cualquier explicación que quiera darse a la eclampsia tardía del puerperio no deja de ser meramente especulativa, a la luz de los conocimientos actuales.

Se ha Ilamado la atención sobre la influencia que pueda tener en su aparición la aplicación o administración de drogas de potencial efecto vasopresor, tales como los derivados del cornezuelo de centeno ( $1-6-7)$. García Rodríguez (2) encontró que el empleo de ocitócicos durante el período del alumbramiento ccasiona una acción hipertensiva entre el 14 y el 26 por ciento de los casos, especialmente cuando se utiliza ergonovina, llegando a elevarse la presión arterial hasta en $60 \mathrm{~mm}$. de $\mathrm{Hg}$. Por el contrario, Michelsen y Guerra (5) advirtieron tan solo discreto aumento de la presión arterial con el empleo de ergonovina, aumento similar al observado en un grupo de pacientes de control. De todas maneras, con su empleo existe la posibilidad de que determinadas pacientes presenten cambios vasculares con efecto presor, especialmente si existe un terreno favorable para ello.

En cuanto hace relación al manejo de este tipo de complicación del puerperio se reduce a una conducta puramente médica, pues ya está descontado el aspecto obstétrico. Lo funda- mental, como en cualquier forma de eclampsia, es detener prontamente el estado convulsivo. Esto se consigue con más facilidad que estando la paciente embarazada, pues es indudable que los factores influyentes en la aparición de la toxemia, tienen que ser más acentuados cursando aún el embarazo. Además, el hecho de que el feto ya no cuente para efecto de las medidas terapéuticas, permite, en caso necesario, utilizar con más liberalidad los agentes anticonvulsivantes Con el mismo esquema de tratamiento que empleamos para la eclampsia intraparto (8) a base de fenobarbital, sulfato de magnesio y acepromazina, en el $17 \%$ de las pacientes reaparecieron las convulsiones, mientras en aquella - la intraparto- iteraron en el $49.5 \%$, Controladas las crisis, los barbitúricos deben suministrarse por vía oral, siquiera durante una semana.

La profilaxis de la eclampsia del puerperio se hace aplicando barbitúricos (fenobarbital $200 \mathrm{mg}$.) en los minutos que siguen al alumbramiento, a toda mujer en quien previamente se comprobaron manifestaciones de toxemia gravídica, continuándolos por vía oral durante los tres primeros días del puerperio. Si se ha seguido esta precaución y si existe una indicación formal, pueden utilizarse a la vez los derivados del cornezuelo de centeno pues así se contrarresta su posible efecto vasopresor.

Tratándose de convulsiones aparecidas tardíamente en el puerperio, ha 
de aceptarse con mucho recelo el diagnóstico de eclampsia. Será necesario un estudio exhaustivo de cada paciente antes de concluir en el diagnóstico de toxemia puerperal, pues la mayoría de esos casos son ocasionados por problemas de tipo vascular, neurológico o metabólico, ajenos por completo al síndrome de preec'ampsia-eclampsia.

Por último, aunque pueda pensarse que la eclampsia del puerperio es menos peligrosa que la forma intravarto, los resultados en este grupo de 61 enfermas indican que la mortalidad materna es sensiblemente igual $(3.1 \%$ y $2.9 \%$ respectivamente).

\section{RESUMEN}

Se presenta un estudio de 64 casos de eclampsia del puerperio observados en el Instituto Materno Infantil, de Bogotá, que corresponden al $14.8 \%$ de todas las eclampsias atendidas en seis años.

En el $90 \%$ de los casos las convulsiones aparecieron en las primeras 24 horas del puerperio. La aparición más tardía fue al noveno día del potsparto. El tratamiento consistió en la aplicacón simultánea de fenobarbital, sulfato de magnesio y acepromazina.

Cuatro mujeres presentaron hemorragia cerebral, de las cuales fallecieros dos $(3.1 \%)$.

\section{BIBLIOGRAFIA}

1. Canillas V.: Eclampsia del puerperio. Rev. Paraguaya Gin. y Obst. 3: 63, 1961.

2. GARCIA M. F.: Efecto tensional de los ocitócicos administrados después del parto. Rev. Obst. Gin. Venezuela, 22: 545, 1962.

3. HARER W. B., Mc INDOE D. W.: Postpartum eclampsia treated by curettage. Am. J. Obst. \& Gynec., 84: 1349, 1962.

4. HOFMEISTER F. J. y BROWN R. C.: Postpartum convulsions. Am. J. Obst. \& Gynec., 65: 498. 1953.

5. MICHELSEN J. y GUERRA A. J.: Efectos cardiovasculares del maleato de ergonovina en el puerperio inmediato. Universitas Médica, 5: 179, 1963.

6. RUBIN A. y ERDE A.: Postpartum eclampsia. Obst. \& Gynec., 24: 448, 1964.

7. SAMUels B.: Postpartum eclamosia.Obst. \& Gynec., 15: 749, 1960.

8. SANCHEZ F.: Eclampsia intraparto. Estudio de 135 casos. Rev. Col. Obst y Gin., 15: 397, 1964.

9. STANDER H. J., BONSNES R. W. y STROMme W. B.: Late postpartum eclampsia. Am. J. Obst. \& Giec., 52: 765, 1946.

10. STEINER G. J. y PLASHA M. K.: Late postpartum eclampsia. Report of a case. Obst. \& Gynec., 24: 594, 1964.

11. TAtum H. J.: Postpartum eclampsia occurring on the fifteenth postpartum day. Am. J. Obst. \& Gynec., 64: 1388, 1952.

12. TATUM H. J.: A new classification of peripartal toxemia. Postpartum phase. Cl. Obst. and Gyn., 5: 670, 1962. 